\title{
Sensitivity of turbulent stresses in boundary layers to cross-wire probe uncertainties in the geometry and calibration procedure
}

\author{
R. Baidya · J. Philip - N. Hutchins · J. P. Monty · I. Marusic
}

Received: date / Accepted: date

\begin{abstract}
The sensitivity of measured turbulent stresses to uncertainties in the probe geometry and calibration procedure is investigated for a cross-wire probe in a turbulent boundary layer using direct numerical simulation (DNS) data. The errors investigated are guided by experiments, and to replicate the full experimental procedure, the cross-wire calibration procedure is simulated to generate a voltage-tovelocity mapping function, which is then utilised to calculate the measured velocity from simulated cross-wire voltages.
\end{abstract}

We show that wire misalignment can lead to an incorrect mean wall-normal velocity and Reynolds shear stress in the near-wall region due to the presence of shear. Furthermore, we find that misalignment in the wire orientation cannot be fully accounted for through the calibration procedure, presumably due to increased sensitivity to an out-of-plane velocity component. This has strong implications if using a generic commercial cross-wire probe, since inclining these probes to gain access to the near-wall region can lead to a large error (up to $10 \%$ ) in turbulent stresses and these errors can manifest in the log region and beyond to half the boundary layer thickness. For uncertainties introduced during the calibration procedure, the Reynolds shear stress is observed to exhibit an elevated sensitivity compared with other turbulent stresses. This is consistent with empirical observations where the repeatability in the Reynolds shear stress is found to be the poorest.

Keywords cross-wire, sensitivity analysis, wall-turbulence

Department of Mechanical Engineering, University of Melbourne, Melbourne, Victoria 3010, Australia

E-mail: baidyar@unimelb.edu.au

\section{Introduction}

In complementary papers (Baidya et al., 2019; Philip et al., $2013 b$ ), we examined the finite sensor dimension effects for a cross-wire probe (hereafter referred to as an $\times$-probe), where we found good agreement between the simulated probe and experiments for the normal stresses, but not for the Reynolds shear stress. These simulations assume an ideal probe and calibration; however, in practice deviations arise from these idealisations due to inherent limitations present during the manufacturing and calibration procedure. Here, the effects of these uncertainties present in the probe geometry, orientation and calibration are explored and we test whether they lead to a closer match between the simulated and experimental profiles.

Among the effects due to the uncertainties in the probe geometry, orientation and calibration, the uncertainties in calibration are most explored in the literature. Each hot-wire is unique and therefore exhibits a distinct mapping function between the velocities and voltages, which needs to be determined individually. This is achieved through a calibration step where the hot-wire is placed in a uniform flow with a known velocity and the output voltages are measured. However, due to the uncertainties associated with the calibration procedure and changes in the wire and ambient properties with time, the obtained mapping function may not correspond to the actual one, leading to an error in the inferred velocity (Talluru et al., 2014). Yavuzkurt (1984) considered typical sources of uncertainty during the calibration of the single normal wire and found that with a well-designed calibration process, the uncertainty level of $1 \%$ and $2 \%$ can be reached for the $U$ and $\overline{u^{2}}$ statistics, respectively. The uncertainty of the hot-wire anemometry system is also investigated by Jørgensen (1996) who, based on uncertainty in the measured velocity during the calibration procedure and subsequent error in fitting a functional form to the velocity- 
voltage map also obtained a value of $1 \%$ and $2 \%$ for the uncertainty in the $U$ and $\overline{u^{2}}$ statistics. Furthermore, Jørgensen (1996) found that while the uncertainty in $U$ and $\overline{u^{2}}$ statistics from the $\times$-probe are comparable to that from the single normal hot-wire, the $\overline{v^{2}}$ and $\overline{w^{2}}$ statistics have elevated uncertainty level of $4 \%$.

Although uncertainties in the probe geometry and orientation can be typically accounted for when calibrating single hot-wire (Segalini et al., 2011), this may not necessarily be the case for an $\times$-probe. This is due to the additional requirement for the $\times$-probe to capture the spanwise and wallnormal velocities (which are less energetic than the streamwise velocity in a wall-bounded flow) and increased uncertainties in the probe geometry and orientation compared to the single hot-wire due to the addition of the second wire. Hence, here we explore the sensitivity of turbulent stresses measured using $\times$-probe to misalignment in the wire position and angular orientation in $\S 3$ and $\S 4$, respectively. In addition, we assess the relative sensitivity of the turbulent stresses measured using $\times$-probe to a non-ideal calibration in $\S 5$.

In this paper, we use the coordinate system $x, y$ and $z$ to refer to the streamwise, spanwise and wall-normal directions, respectively; while $u, v$ and $w$ are the corresponding fluctuating velocities. Furthermore, capitalisation and overbars indicate time-averaged quantities, while superscript ' + ' denotes normalisation by viscous units (e.g., $U^{+}=U / U_{\tau}$, $z^{+}=z U_{\tau} / v$, where $U_{\tau}$ and $v$ correspond to the mean friction velocity and kinematic viscosity, respectively).

\section{Methodology}

\subsection{Direct numerical simulation database}

In order to simulate $\times$-probe in a wall-bounded flow a Direct Numerical Simulation (DNS) channel flow database from del Álamo et al. (2004) at a friction based Reynolds number $R e_{\tau}=934$ is used, so that the measured velocity is constructed from a realistic velocity field. The DNS database has a domain size of $8 \pi h, 3 \pi h$ and $2 h$, where $h$ denotes the half channel height, in the $x, y$ and $z$ directions respectively. A Fourier, Fourier and Chebyshev spatial discretization scheme is used in $x, y$ and $z$ directions, with a grid size corresponding to $3072 \times 2304 \times 385$. The available database has been de-aliased and interpolated at a grid resolution of $\Delta x \times \Delta y \approx 7.6 \times 3.8$ viscous units, while $\Delta z$ varies from 0.03 at the wall to 7.6 viscous units at the channel centreline.

\subsection{Modelling the finite sensor effects}

The hot-wires are modelled as box-filters with length $l$, equal to the sensor length, which have been shown to capture the

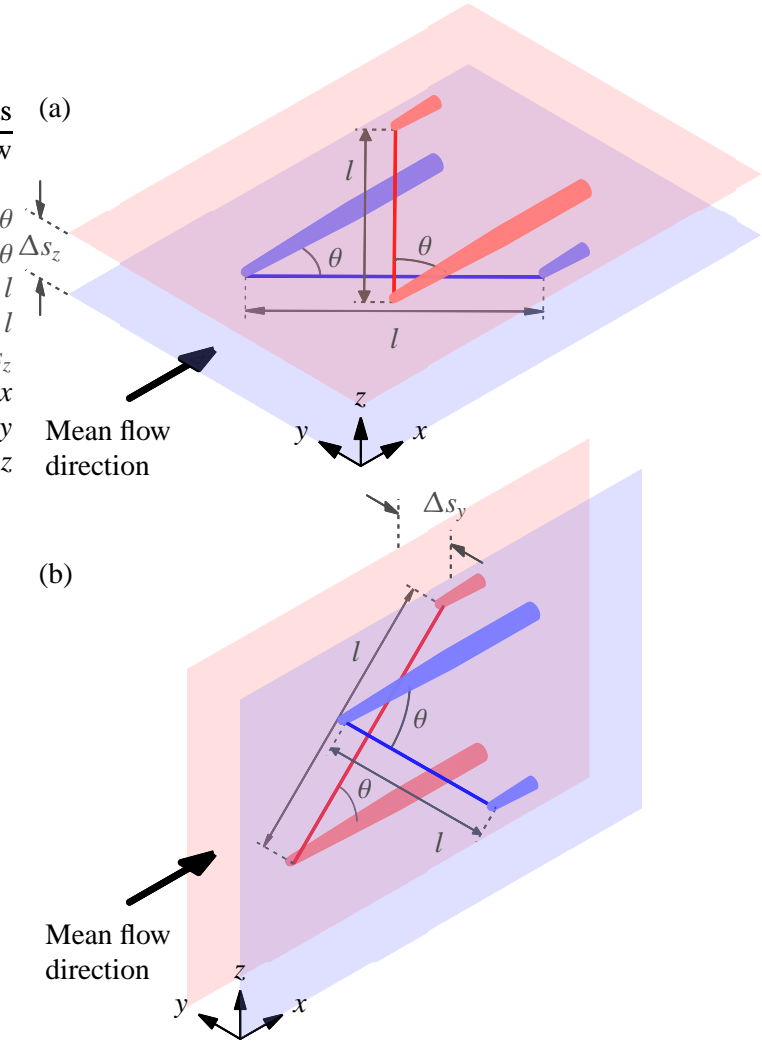

Fig. 1: $\times$-probe configured to measure (a) $u$ and $v$ velocities and (b) $u$ and $w$ velocities. The red and blue wires are located on the respectively coloured planes, which are separated by $\Delta s_{y}$ and $\Delta s_{z}$ in the $y$ and $z$ directions for the two configurations, while $l$ and $\theta$ denote the wire length and wire angle, respectively.

$\overline{u^{2}}$ results from hot-wire experiments fairly well (Chin et al., 2011; Philip et al., 2013a). Furthermore, Örlü \& Schlatter (2013) report good agreement for the higher-order moments, probability density functions and energy spectra between hot-wire experiments and DNS data where spanwiseaveraging over a viscous-scaled hot-wire length, $l^{+}$, has been performed. Here, we take the hot-wire output voltage to be only sensitive to the velocity component normal to the wire since the tangential cooling has a negligible contribution to the total heat convention that drives the hot-wire output (Champagne et al., 1967). Thus, the wire-normal component is evaluated along the hot-wire sensor from the DNS velocity field and then filtered. Consequently, the resulting filtered wire-normal velocity from the simulated probe has a one-to-one relationship with the hot-wire output voltage. Note that here, we have neglect interference from supporting prongs which are unavoidable in experiments. However, we have endeavoured to minimise the prong interference effects in the experiments by maintaining a length-to-diameter ratio in excess of 200 for the hot-wire (Ligrani \& Bradshaw, 1987) and the prong diameter to be comparable to the outer 
diameter of an unetched Wolloston wire. Hence, we expect the probe interference effects to be minimal compared to the errors introduced due to probe misalignment and non-ideal calibration procedure investigated here. For the study, two $\times$-probe configurations used to measure the $v$ and $w$ velocities in addition to $u$, as shown in figure $1(a)$ and $(b)$, are considered. Furthermore, the two configurations are hereafter referred to as a $u-v$ and $u-w \times$-probe, respectively. Here, $l, \theta$ and $\Delta s$ denote the wire length, wire angle and separation between the wires. Throughout the paper, the dimension of the simulated $\times$-probe is maintained at $l^{+}=20, \theta=45^{\circ}$ and $\Delta s^{+}=7$ in the $y$ or $z$ direction. These dimensions are based on typical $\times$-probes built at the University of Melbourne to study high Reynolds number turbulent boundary layer flows. Note that a change in the wire length, wire angle or wire separation distance lead to a modification in the dimensions of the $\times$-probe, influences of which are reported in the complementary papers (Baidya et al., 2019; Philip et al., 2013b).

\subsection{Modelling the hot-wire calibration}

The usual procedure when simulating an $\times$-probe is to obtain an explicit expression for the relationship between the filtered wire-normal velocities from the two wires in the $\times$ probe and the measured velocities (Moin \& Spalart, 1989). However, in experiments it is challenging to determine such explicit relationships due to departures from idealistic scenarios considered when formulating the explicit expression. Instead in the experiments, the voltages from $\times$-probe is tabulated at different $U$ and $V$ (or $W$ ) velocities in an irrotational flow to determine the voltage-to-velocity mapping function. Thus, the calibration should account for any imperfections in the $\times$-probe geometry due to uncertainties present during the fabrication stage, provided that the measurement is conducted in an irrotational flow. However, wallbounded flows are anisotropic with a presence of strong shear in the near-wall region, which leads to an error that cannot be corrected by calibration. It turns out that these errors can be exasperated by imperfections present in the $\times$-probe geometry (a detailed discussion is provided in $\S 3$ and 4). Here, we investigate these errors using the DNS database, which allows the probe geometry to be precisely specified.

In order to simulate the experimental calibration procedure, a functional form relating the output hot-wire voltage to the wire-normal velocity is required. Here we use the unmodified King's law (King, 1914), where the constants are chosen based on theoretical values obtained for an idealised hot-wire and anemometry system (Perry, 1982). It should be noted that the exact functional form used is not critical, and any relationship that captures the typical hot-wire characteristics, namely an increased sensitivity at lower velocity (Bruun, 1995) would work equally well. Furthermore, for the current work, the full-scale voltage range is set at

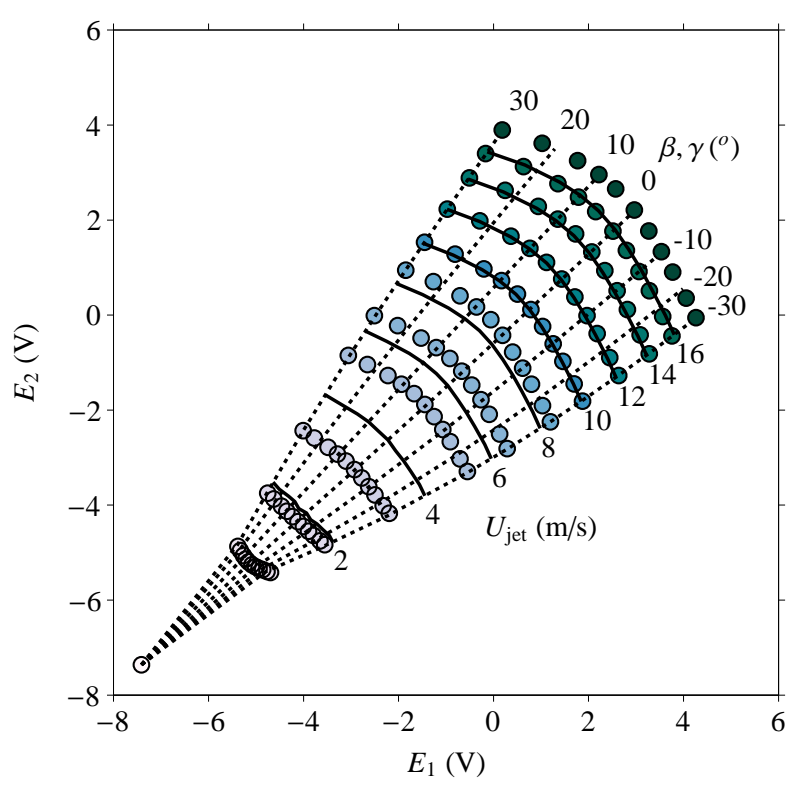

Fig. 2: Typical two-dimensional calibration showing voltage pair $E_{1}, E_{2}$. $\bigcirc$ Calibration points, — constant jet velocity lines and $\cdots$ constant jet angle lines determined from calibration points. The symbols are coloured according to the jet velocities.

approximately $15 \mathrm{~V}$, as in the experiments conducted at the University of Melbourne (e.g., figure 2).

For the experiments, the $\times$-probe is calibrated in a potential core of a jet flow, and the jet velocities and the yaw (or pitch) angle between the jet and $\times$-probe is varied to specify the $U$ and $V$ (or $W$ ) velocities. Figure 2 shows voltage pair $E_{1}$ and $E_{2}$ from two hot-wires, at discrete sets of flow velocities and flow angles following a typical calibration procedure in the experiments. Note, here $E_{1}$ and $E_{2}$ corresponds to the amplified output voltage from the anemometer system and not the voltage at the top of the bridge in the anemometer circuit. Hence, for the simulated calibration procedure, the hot-wire voltages are also tabulated at similar sets of flow velocities and flow angles. Here, we assume as in the experiments that the voltage-velocity relation exhibited by the hot-wire holds equally well across the boundary layer flow. However, this does not necessarily hold when the hot-wire is in close proximity to the wall (e.g., Janke, 1987). Hence, we limit our analysis to the $z^{+}>10$ region, where $U$ profiles from differing wall thermal characteristics show collapse (Hutchins \& Choi, 2002), and therefore we expect minimal influence of the wall on the hot-wire voltage-velocity relation in this region.

\section{$2.4 \times$-probe simulation procedure}

Figure 3 summarises the $\times$-probe simulation procedure used in the current investigation. Here, the hot-wire model combines the finite sensor effects and the King's law relationship 
Calibration

Known velocities $\mathbf{U}$ and $\mathbf{V}$

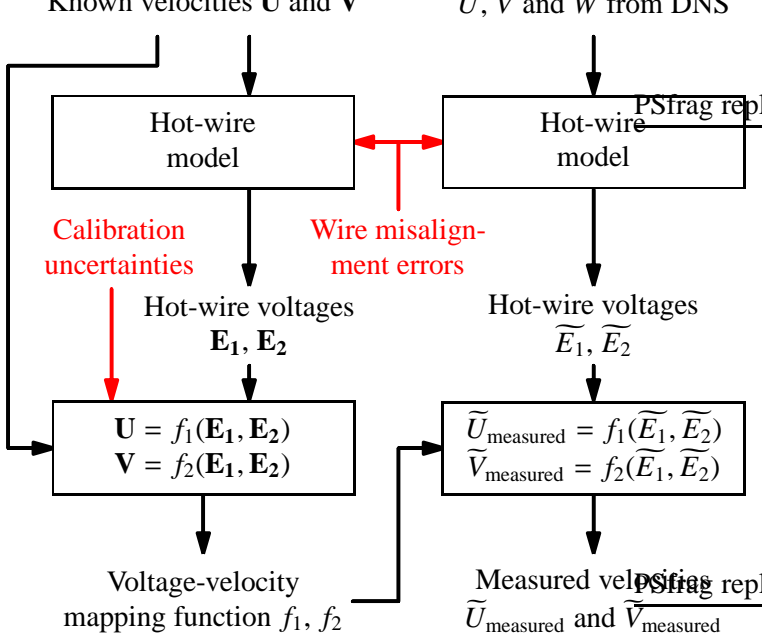

Measurement

$\widetilde{U}, \widetilde{V}$ and $\widetilde{W}$ from DNS

$\widetilde{U}_{\text {measured }}$ and $\widetilde{V}_{\text {measured }}$
Fig. 3: Schematic showing the $\times$-probe simulation procedure. The tilde is used to denote the total quantity, while the bold symbol indicate a matrix with multiple entries. The error sources investigated are shown in red.

between the hot-wire voltages and the wire-normal velocities discussed in $\$ 2.2$ and 2.3. Hence, for the simulated calibration procedure, the model is applied for each of the hotwires in the $\times$-probe to obtain voltages $\mathbf{E}_{\mathbf{1}}$ and $\mathbf{E}_{\mathbf{2}}$ from calibration velocities $\mathbf{U}$ and $\mathbf{V}$ (or $\mathbf{W}$ depending on the $\times$-probe configuration). Note that the bold symbols indicate matrices, where each entry is unique in $U-V$ space. This allows construction of voltage-to-velocity mapping function $f_{1}$ and $f_{2}$ from the tabulated values. Similar to calibration procedure, the total velocities $\widetilde{U}, \widetilde{V}$ and $\widetilde{W}$ (here tilde is used to denote the total quantities) from DNS is supplied to the model to simulate hot-wire voltages $\widetilde{E_{1}}$ and $\widetilde{E_{2}}$ obtained in a channel flow, which are then converted to measured velocities, $\widetilde{U}_{\text {measured }}$ and $\widetilde{V}_{\text {measured }}$, from the simulated $\times$-probe. Thus, the differences between the measured and original velocity (e.g., $\widetilde{U}_{\text {measured }}-\widetilde{U}$ ) correspond to errors introduced from the use of $\times$-probe. Furthermore, the wire misalignment scenarios examined in $\S 3$ and 4 effects the hot-wire model, while calibration errors investigated in $\S 5$ relate to uncertainties in the generation of the mapping function, and these error sources are shown in red in figure 3.

\section{The effects of misalignment in the wire position}

Due to the uncertainties present during the manufacturing process, differences will always exist between the actual dimension of the $\times$-probe built, compared to the specified design. For example, the microscope used by the authors to measure the wire length, $l$, had a resolution of $20 \mu \mathrm{m}$, leading to an error of $\pm 5 \%$ when $l=0.5 \mathrm{~mm}$. For the same reasons, the location where the two wires cross and the indi-

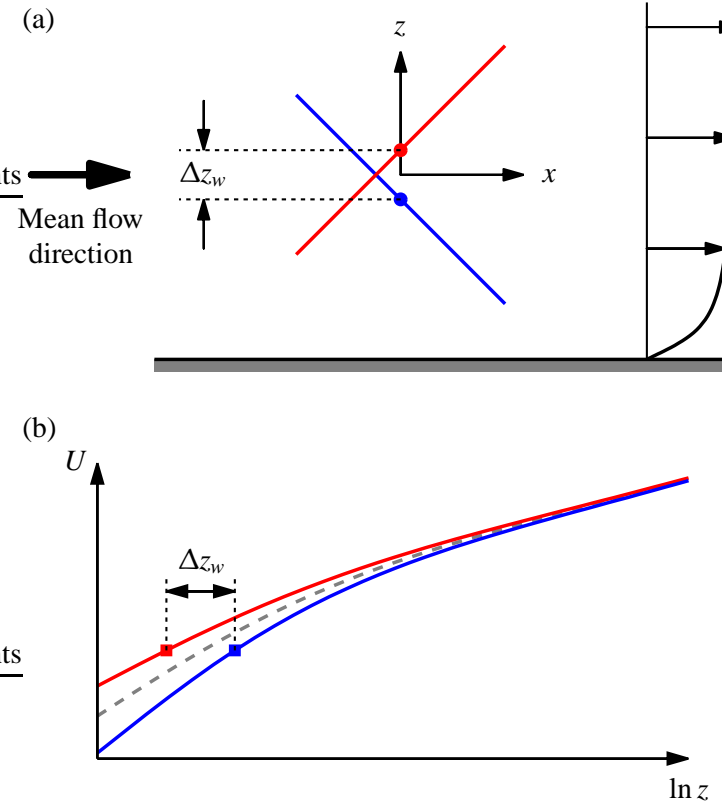

Fig. 4: Wall-normal misalignment between the two wires. (a) Side view of a $u$-w $\times$-probe with a (positive) wall-normal misalignment $\Delta z_{w}$, where the centre of the wires are denoted in circles. (b) The local mean velocity at the centre of the wires, where the square symbols correspond to the location with an equivalent local velocity. The dashed line indicates the original statistic.

vidual centres of each wires did not coincide, as illustrated in figure 4(a), for a typical $\times$-probe used in the experiments. In addition, the hot-wire locations may be altered due to aerodynamic effects and hence the $\times$-probe geometry in a flow during the experiment may differ from that measured using the microscope without presence of a flow.

To assess the misalignment in the wall-normal direction between the centre of the two wires centres, $\Delta z_{w}$, the hotwires in $\times$-probe can be considered as two individual slanted wires. Then $U$ statistics from each slanted wires can be calculated using the individual calibration curves at the zero angle calibration (determined from a polynomial fit to the hot-wire voltage, $E$, versus $U$ relationship). Thus, $\Delta z_{w}$ can be estimated from the offset in the $z$ direction required to collapse the individual mean velocity profile as illustrated in figure $4(b)$. Using this method, we find that the typical uncertainty associated with the placement of wire centres on the manufactured $\times$-probe to be $\pm 30 \mu \mathrm{m}$, which corresponds to $\left|\Delta z_{w}^{+}\right| \approx 2$.

Figure 5 shows deviations in the measured wall-normal mean velocity and Reynolds shear stress for a wall-normal misalignment corresponding to $\left|\Delta z_{w}^{+}\right|=2$ (symbols) between the two wires for the $u-w \times$-probe. A clear deviation in $W$ and $\overline{u w}$ from $\Delta z_{w}^{+}=0$ (lines) in the near-wall region is evident. This deviation occurs due to the presence of strong shear near the wall, leading to different local mean velocity 

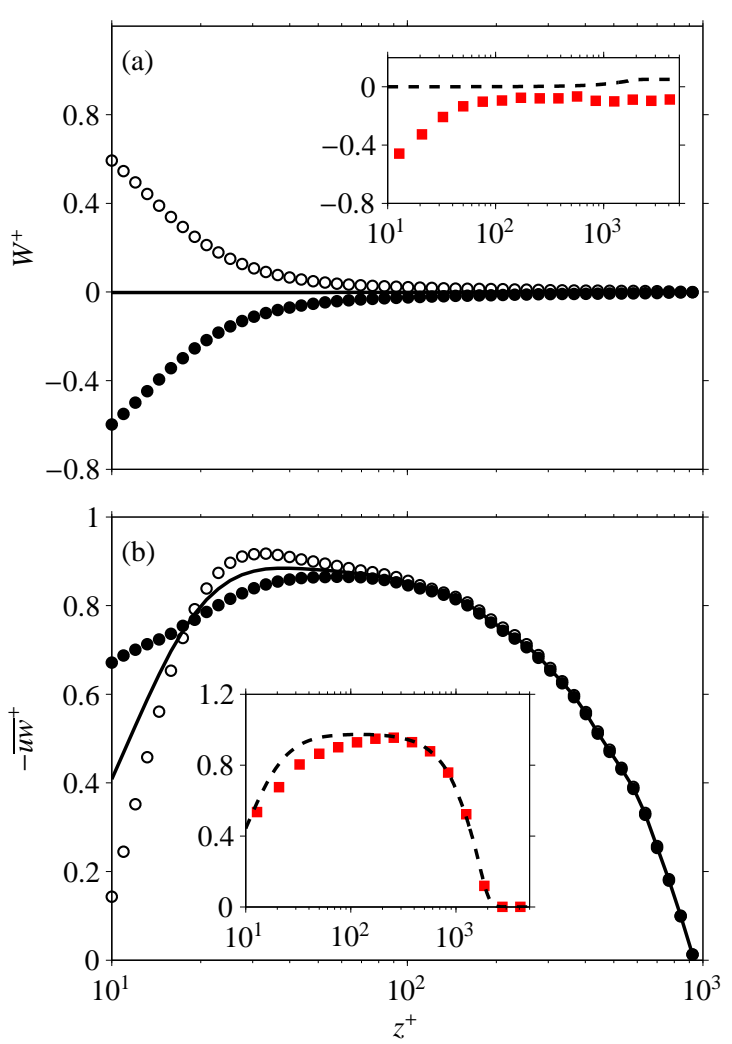

Fig. 5: Sensitivity of a $u-w \times$-probe to the wall-normal misalignments between the two wires. The measured (a) wallnormal mean velocity and (b) Reynolds shear stress. The solid line corresponds to a probe without any misalignment, while the symbols correspond to wire misalignment of two viscous units in the $z$ direction $\left(\left|\Delta z_{w}^{+}\right|=2\right)$. The two symbols correspond to scenarios where the wire closest to the wall is inclined at $-45^{\circ}$ and $\bigcirc 45^{\circ}$ with respect to the $x$ direction, respectively. (Insets) Comparisons of experimental ( $\square$ ) and original DNS (-- ) statistics at $R e_{\tau} \approx 2,500$ in a turbulent boundary layer.

experienced by each wire due to $\Delta z_{w} \neq 0$ (a similar mechanism lead to $V \neq 0$ for $\times$-probe configured to measure the $u$ and $v$ velocities (Philip et al., 2013b)). It should be noted that rest of the turbulent stresses and $U$ remain insensitive to the misalignment errors (also observed by Talamelli et al. (2000)) and are not reported here. Furthermore, for each wall-normal misalignment, two configurations are possible depending on the inclination angle of the wire closest to the wall and are denoted by the solid and empty symbols when the wire closest to the wall is inclined at $-45^{\circ}$ or $45^{\circ}$. Since the statistics for the solid symbols is a better representation of the experimental results for both the $W$ and $\overline{u w}$ statistics compared to the solid lines (see insets), we postulate that for the $\times$-probe used for this particular experiment, the wire inclined at $-45^{\circ}$ is positioned closer to the wall than the other wire.
Although not shown here, a wall-normal misalignment $\Delta z_{w}$, between the two wires also leads to a non-zero $V$ to be recorded in the near-wall region when using a $\vee$-probe, due to a similar reason. For a $u-v \times$-probe, the wires are placed at two different $z$ locations by design, which again leads to a non-zero $V$ in the near-wall region (Philip et al., $2013 b$ ). Furthermore, typically the wire spacing in the $u$ $v \times$-probe far exceeds the wire misalignment, and thus the additional errors introduced by wire misalignment remain insignificant.

\section{The effects of misalignment in the wire angular orientation}

One of the challenges working with sub-miniature probes is ensuring they are at a correct angular orientation with respect to the wind-tunnel frame of reference the velocity measurements are based on. Although the alignment of the $x$-probe is checked with the aid of a microscope and a digital camera equipped with a long-focus lens prior to measurements, due to the highly magnified view, accurate determination of the wind-tunnel $x-y-z$ coordinate system within the field of view is difficult. Since this procedure is required each time the wires are repaired, the sensitivity of the measured turbulent stresses to misalignment in angular orientation will influence the degree to which the statistics can be reproduced. While it has been demonstrated that for single hot-wire probes the influence for angular misalignment is minimal for the $z^{+}>20$ region when the misalignment is less than $5^{\circ}$ (Segalini et al., 2011), the authors are unaware of such studies for the $\times$-probes. Hence, the sensitivity of the measured turbulence intensities using $\times$-probes to deviation in the angular orientation from the ideal case is quantified utilising the DNS velocity fields. This is achieved by rotating the sensing element of the simulated $\times$-probes in the $x, y$ and $z$ directions (corresponding to angles $\alpha, \beta$ and $\gamma$ ) and examining the turbulence statistics calculated. We note that, here the probe misalignment is accounted for (through a simulated calibration procedure) when obtaining a mapping function used to evaluate the velocities and therefore, these errors are separate from biases that can be introduced due to a non-ideal calibration procedure, which are reported in $\S 5$.

Figure 6 shows the effect on the measured turbulent stresses using the $u-v \times$-probe, at misalignment of $\pm 10^{\circ}$ in $\alpha, \beta$ and $\gamma$. Although a $\pm 10^{\circ}$ deviation from the desired orientation may seem excessive, this is a reasonable upper bound considering the probe body can be deliberately inclined to allow access to the near-wall region leading to $\beta \neq 0$. Note that, due to filtering effect from a finite sized $\times$-probe, differences exist in the turbulent stress profiles calculated from the original DNS (-- -) and that recorded by an ideally aligned probe (-) (Suzuki \& Kasagi, 1992). However, the spa- 

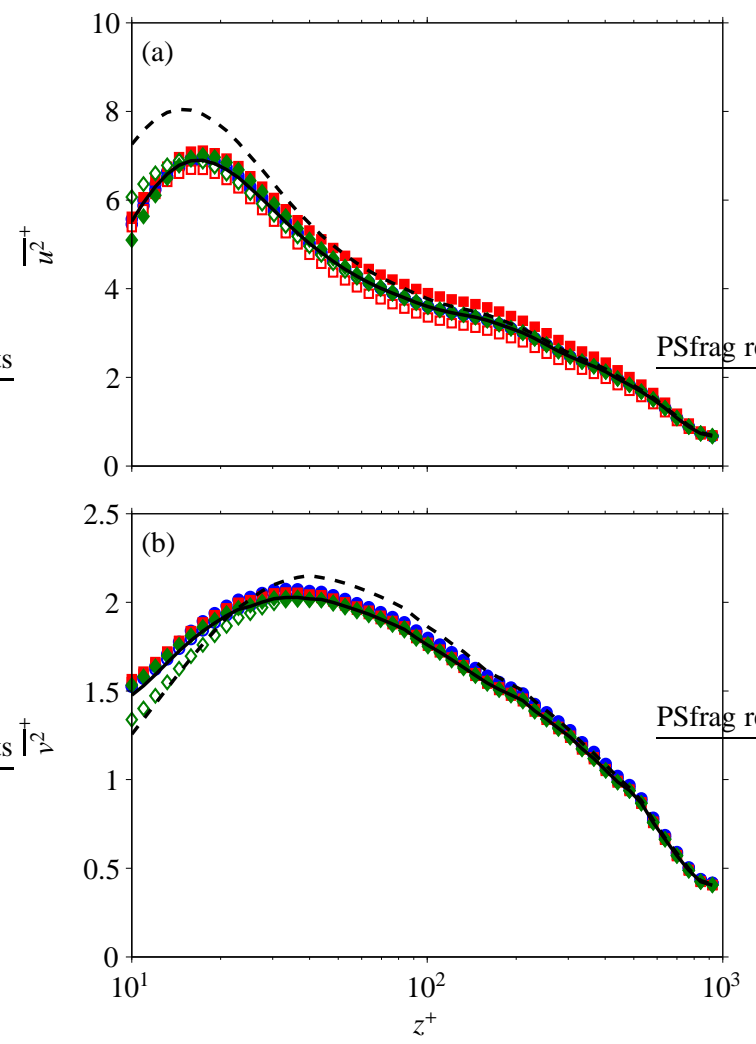

Fig. 6: Sensitivity of a $u$-v $\times$-probe $\left(l^{+}=19, \Delta s_{z}^{+}=7\right)$ to angular misalignment. (a) Streamwise and (b) spanwise variances. - - Original DNS statistic, $-\times$-probe in an ideal alignment $\left(\alpha_{\text {mis }}, \beta_{\text {mis }}, \gamma_{\text {mis }}=0^{\circ}\right) . \times$-probe with an angular misalignment of $\bigcirc \alpha_{\text {mis }}=-10^{\circ}, \bigcirc \alpha_{\text {mis }}=10^{\circ} ; \square \beta_{\text {mis }}=$ $-10^{\circ}, \square \beta_{\mathrm{mis}}=10^{\circ}$; and $\diamond \gamma_{\mathrm{mis}}=-10^{\circ}, \gamma_{\mathrm{mis}}=10^{\circ}$.

tial resolution remains constant between the misaligned and ideally aligned probes, and hence the influence from the misalignment can be isolated by comparing these two cases. One of the advantages of customising probes (as in this study) is that the non-zero probe inclination angle can be accounted for when designing the sensing elements, such that they are at the specified configuration even when the probe inclination angle is non-zero. In contrast, this flexibility is not present in the commercially available probes due to their generic design.

The $u$ - $v \times$-probes show a substantial difference in the measured $\overline{u^{2}}$ statistics throughout the entire $z$ location as shown in figure $6(a)$, when the sensing elements are misaligned about the $y$ axis (shown as squares). The empty and solid symbols are used to denote misalignment in the opposing directions, and this convention is continued throughout the section. Compared to the errors in the $\overline{u^{2}}$ statistics, the error in the $\overline{v^{2}}$ statistics, is limited to measurements in the near-wall region as shown in figure $6(b)$. Contrary to the effects of misalignments in the $\beta$ direction, both $\overline{u^{2}}$ and $\overline{v^{2}}$ are robust to the misalignments about the $x$ ( $\alpha_{\text {mis }}$, circles) and $z$
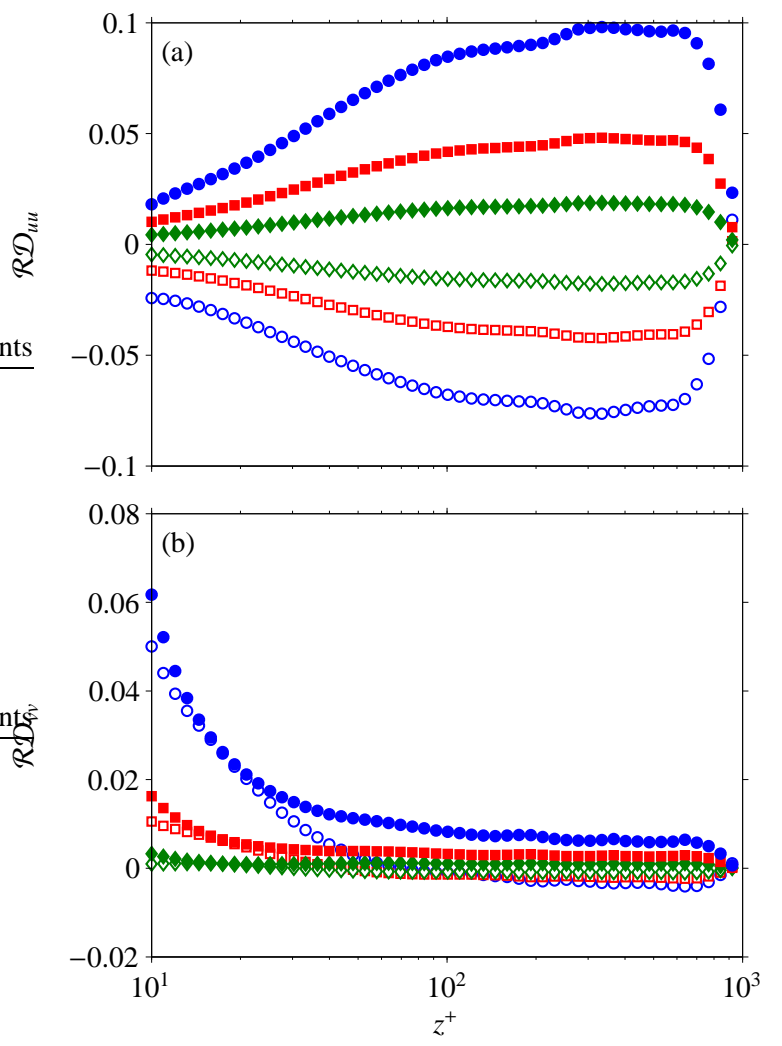

Fig. 7: Relative difference of $u-v \times$-probes $\left(l^{+}=19, \Delta s_{z}^{+}=\right.$ 7) with angular misalignment about the $y$ direction. (a) Streamwise and (b) spanwise variances. Angular misalignment of $\bigcirc \beta_{\text {mis }}=-10^{\circ}, \square \beta_{\text {mis }}=-5^{\circ}, \diamond \beta_{\text {mis }}=-2^{\circ}, \diamond \beta_{\text {mis }}=2^{\circ}$, - $\beta_{\text {mis }}=5^{\circ}$ and $\bullet \beta_{\text {mis }}=10^{\circ}$.

( $\gamma_{\text {mis }}$, diamonds) axes for the $z^{+}>20$ region, as shown in figure 6.

As the effect of misalignment about the $x$ and $z$ axes were found to be insignificant (see figure 6), further investigation is only conducted for the effect of misalignment about the $y$ axis. Thus, additional simulations corresponding to cases with misalignment of $\beta_{\text {mis }}= \pm 2^{\circ}$ and $\pm 5^{\circ}$ are performed, and the resulting errors in the $\overline{u^{2}}$ and $\overline{v^{2}}$ are shown as the relative difference in figures $7(a)$ and $(b)$. Here, the relative difference, $\mathcal{R D}$, is defined as

$\mathcal{R} \mathcal{D}_{u_{i} u_{j}}=\frac{\overline{u_{i} u_{j}}-\left.\overline{u_{i} u_{j}}\right|_{\text {ideal }}}{\left.\overline{u_{i} u_{j}}\right|_{\text {ideal }}}$

where, $\left.\overline{u_{i} u_{j}}\right|_{\text {ideal }}$ are the turbulent stresses from the ideally aligned $\times$-probe (i.e., $\alpha_{\mathrm{mis}}, \beta_{\mathrm{mis}}, \gamma_{\mathrm{mis}}=0$ ). Figure $7(a)$ suggests that inclining the probe about the $y$ axis by $10^{\circ}$ to allow near-wall access can lead to $\sim 10 \%$ error introduced for the $\overline{u^{2}}$ statistics. Notably, angular misalignment of a $u-v$ $\times$-probe can lead to an amplified $\overline{u^{2}}$ for positive $\beta$, contrary to finite dimension effects where attenuation of $\overline{u^{2}}$ occurs (Philip et al., 2013b). Furthermore, while strong influence of finite dimension effects is limited to the near-wall region 

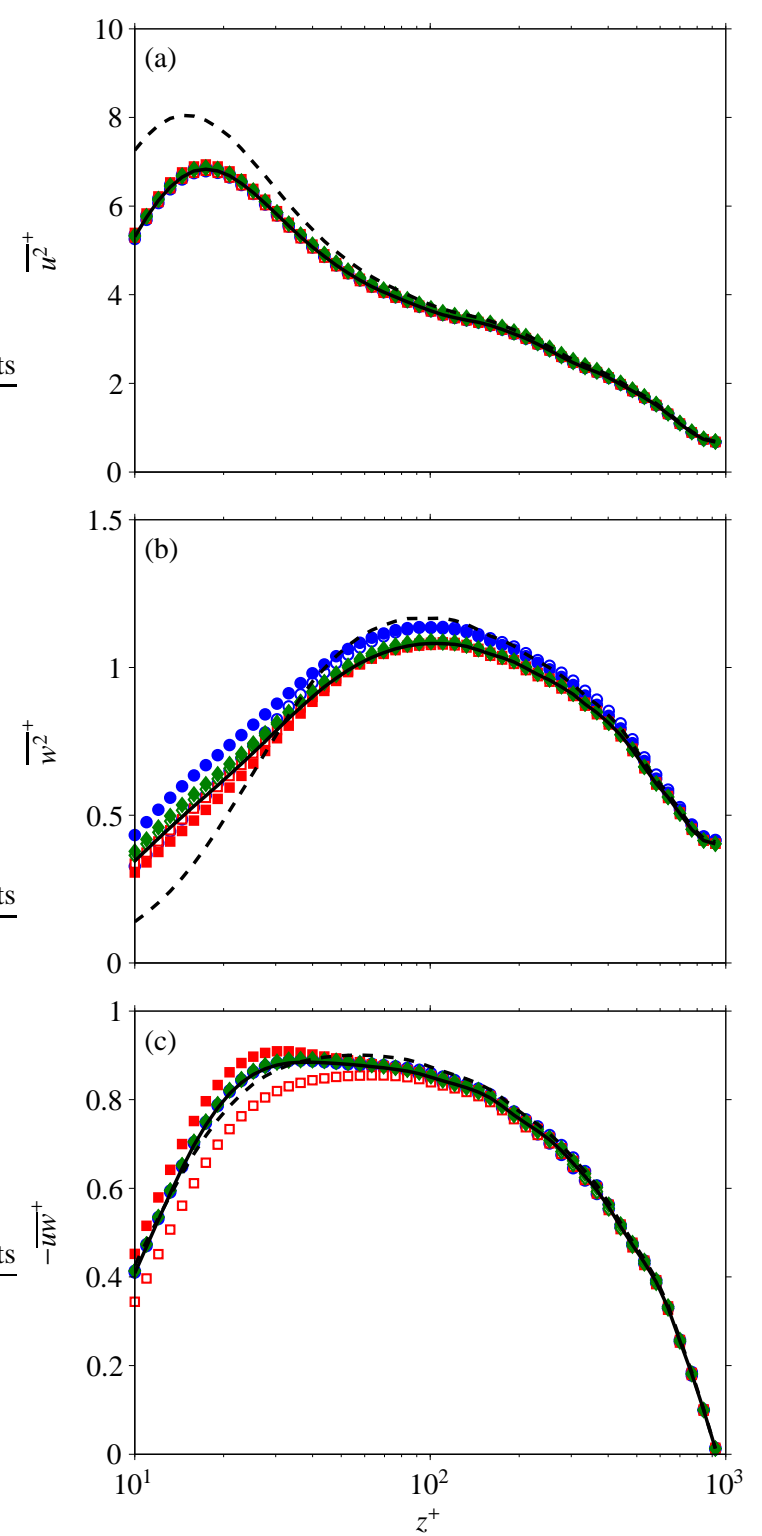

Fig. 8: Sensitivity of a $u$-w $\times$-probe $\left(l^{+}=19, \Delta s_{y}^{+}=7\right)$ to angular misalignment. (a) Streamwise and (b) wall-normal variances, (c) Reynolds shear stress. - - - Original DNS statistic, $-\times$-probe in an ideal alignment $\left(\alpha_{\text {mis }}, \beta_{\text {mis }}, \gamma_{\text {mis }}=0^{\circ}\right)$. $\times$-probe with an angular misalignment of $\bigcirc \alpha_{\text {mis }}=-10^{\circ}$, $\alpha_{\text {mis }}=10^{\circ} ; \square \beta_{\text {mis }}=-10^{\circ}, \square \beta_{\text {mis }}=10^{\circ}$; and $\diamond \gamma_{\text {mis }}=-10^{\circ}$, $\checkmark \gamma_{\text {mis }}=10^{\circ}$.

(e.g., $z^{+}<100$ for the solid and dashed lines in figure 6), the errors due to angular misalignment can remain significant in the $\log$ region and beyond up to $z<0.5 \delta$, which is typically the region of interest in high $R e$ flows (Smits et al., 2011).

Figures $8(a)-(c)$ show the measured $\overline{u^{2}}, \overline{w^{2}}$ and $\overline{u w}$ statistics using a $u-w \times$-probe which has been misaligned about the $x$ (circles), $y$ (squares) and $z$ (diamonds) axes by $\pm 10^{\circ}$. The $\overline{u^{2}}$ statistics from the $u-w \times$-probe are robust to the misalignment errors in all three directions, unlike the $u-v \times$ - probe. Overestimation of the $\overline{w^{2}}$ statistics across entire $z$ location is observed from angular misalignment about the $x$ axis, while no significant change is observed for misalignments about the $y$ and $z$ axes. Finally, the measured Reynolds shear stress is influenced by misalignment in the $\beta$ direction, while it is robust to misalignment in the other two directions. Therefore, simply inclining a $u-w \times$-probe, without accounting for the misalignment of the sensing elements, will lead to incorrect $\overline{u w}$ statistics, particularly in the near-wall region.

From figure 8, we can conclude that probe misalignment about the $x$ and $y$ axes is the primary source of error in the $\overline{w^{2}}$ and $\overline{u w}$ statistics respectively, with little contribution from misalignment in the other direction. Additional cases corresponding to $\alpha_{\text {mis }}= \pm 2^{\circ}, \pm 5^{\circ}$; and $\beta_{\text {mis }}= \pm 2^{\circ}, \pm 5^{\circ}$ are shown in figures $9(a)-(d)$. For misalignment errors corresponding to $\pm 2^{\circ}$, while the uncertainty in the $\overline{w^{2}}$ and $\overline{u w}$ statistics is non-significant in the log region, the error in the near-wall region can be as high as $3 \%$.

The misalignment between the sensor plane of the $x$ probe and the $x-y$ or $x-z$ planes lead to an increased sensitivity to the out-of-plane component. However, the out-ofplane velocity component is not accounted for by a standard $\times$-probe calibration procedure replicated in this study. Hence, the variation in the strength of the out-of-plane component across the boundary layer and between the $u$ - $v$ and $u$ $w \times$-probes may explain why the errors shown in figures 6-9 is dependent on $z$ and the probe configuration. Moreover, the increased sensitivity to the out-of-plane component can lead to a pronounced departure from an idealised hot-wire behaviour relation assumed in many calibration procedure (e.g., effective angle (Browne et al., 1988) and total stress (Zhao et al., 2004) methods), and therefore care should be taken when selecting these procedures. To mitigate some of these errors, we have opted to design our custom sub-miniature $\times$-probes used in the experiments such that the hot-wires remain in parallel with the $x-y$ and $x$-z planes, even when the probe support is inclined by $10^{\circ}$ along the $y$ axis to allow near-wall access. However, in practice a small misalignment still exists due to uncertainty present when fabricating and mounting the $\times$-probes. In fact, the Reynolds shear stress profile in the near-wall region $\left(z^{+}<100\right)$ from the experiments (e.g., figure $5 b$ inset) is underestimated, closely resembling the results from the simulated $\times$-probe with a negative value of $\beta_{\text {mis }}$. Thus, we postulate that a small negative $\beta_{\text {mis }}$ misalignment is still present in this particular probe, leading to a series of measurements from the same probe all showing the near-wall underestimation in the $-\overline{u w}$ statistics (Baidya et al., 2017). For the $\overline{u^{2}}, \overline{v^{2}}$ and $\overline{w^{2}}$ stresses, the influence from misalignment in $\beta$ is small and the finite dimensions effects dominate the stresses measured in experiments. 

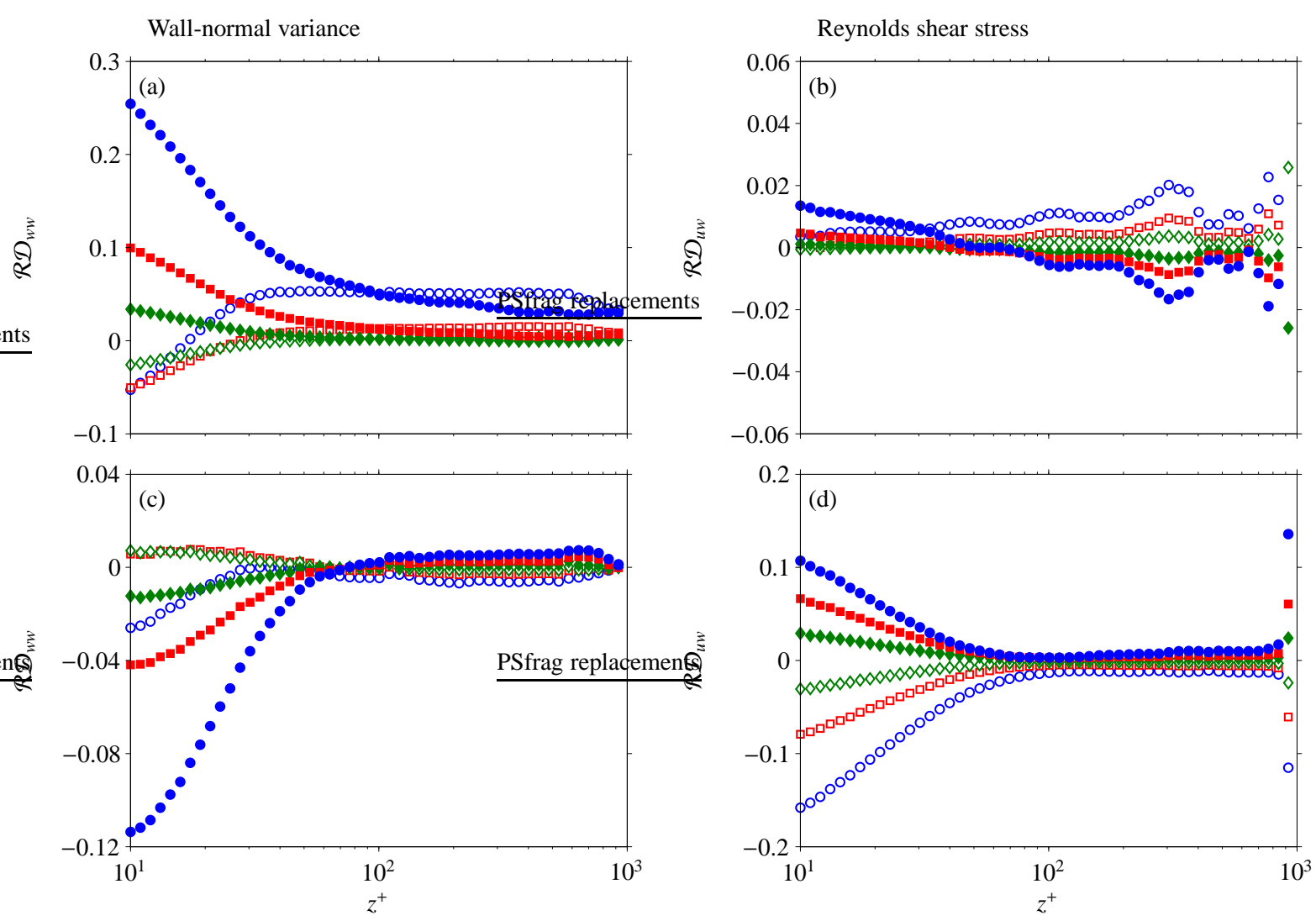

Fig. 9: Relative difference of a $u$-w $\times$-probe with angular misalignment about the $x$ and $y$ direction. Left column (a,c): wall-normal variances, right column (b,d): Reynolds shear stress. Top row (a,b): angular misalignment of $\bigcirc \alpha_{\text {mis }}=-10^{\circ}$, $\square \alpha_{\text {mis }}=-5^{\circ}, \diamond \alpha_{\text {mis }}=-2^{\circ}, \diamond \alpha_{\text {mis }}=2^{\circ}, \square \alpha_{\text {mis }}=5^{\circ}$ and $\bullet \alpha_{\text {mis }}=10^{\circ}$; bottom row (c,d): angular misalignment of $\bigcirc$ $\beta_{\mathrm{mis}}=-10^{\circ}, \square \beta_{\mathrm{mis}}=-5^{\circ}, \diamond \beta_{\mathrm{mis}}=-2^{\circ}, \diamond \beta_{\mathrm{mis}}=2^{\circ}, \nabla \beta_{\mathrm{mis}}=5^{\circ}$ and $\bullet \beta_{\mathrm{mis}}=10^{\circ}$.

\section{The effects of non-ideal calibration}

In the simulation study conducted in $\S 3$ and 4 , a perfect calibration is assumed where no errors in the measured velocity or probe alignment occur. In reality, however, the calibration process is not perfect, since the orientation of the calibration device, the measured voltages and the supplied velocities each have associated uncertainties which lead to an error in the produced calibration map. Here, we investigate how the errors propagate from the calibrated to the measured velocity and determine their sensitivity to perturbations in the voltage-to-velocity mapping function.

\subsection{Propagation of errors}

Suppose that the $u$ velocity can be fully expressed in terms of measured variables $q_{1}, q_{2}, \ldots, q_{n}$ as

$u=f\left(q_{1}, q_{2}, \ldots, q_{n}\right)$.

Then, errors in the velocity from perturbations to $q_{1}, q_{2}, \ldots, q_{n}$ are given by

$\mathcal{E}_{u}=f\left(q_{1}+\mathcal{E}_{q_{1}}, q_{2}+\mathcal{E}_{q_{2}}, \ldots, q_{n}+\mathcal{E}_{q_{n}}\right)-f\left(q_{1}, q_{2}, \ldots, q_{n}\right)$, where $\mathcal{E}$ is used to represent a perturbation to the variable indicated in the subscript. Using Taylor expansion, equation (3) can be expressed as

$$
\begin{aligned}
\mathcal{E}_{u}=\frac{\partial f}{\partial q_{1}} \varepsilon_{q_{1}}+\frac{\partial f}{\partial q_{2}} \varepsilon_{q_{2}} & +\ldots+\frac{\partial f}{\partial q_{n}} \varepsilon_{q_{n}} \\
& +O\left(\mathcal{E}_{q_{1}}^{2}\right)+O\left(\mathcal{E}_{q_{2}}^{2}\right)+\ldots+O\left(\mathcal{E}_{q_{n}}^{2}\right) .
\end{aligned}
$$

5.2 The sensitivity to misalignment errors when performing two-dimensional calibration

The $\times$-probe used to obtain the experimental statistics shown in figure 5(a) and $(b)$ insets are calibrated in situ using a jet calibration facility. This procedure allows the $\times$-probe to be calibrated without the need to unmount the probe from the traverse, avoiding misalignment of the probe between calibration and measurement. However, to calibrate, the jet is placed inside the wind tunnel working section, and hence each calibration requires the jet to be aligned with respect to the wind tunnel, which requires some degree of visual inspection. Therefore, misalignment may exist between the jet and the wind-tunnel frame of reference, that is the $x$ coordinate during the calibration may not align with the flow di- 
(a)

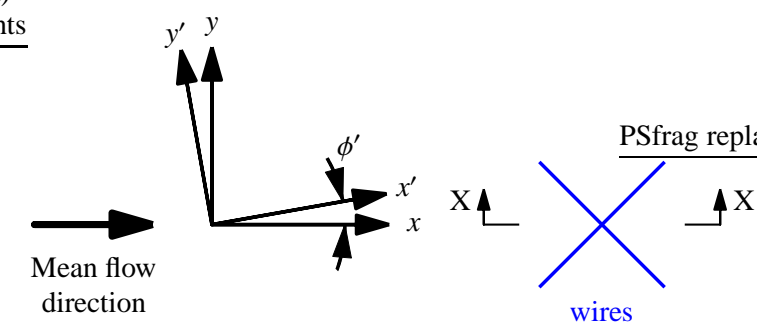

(b)

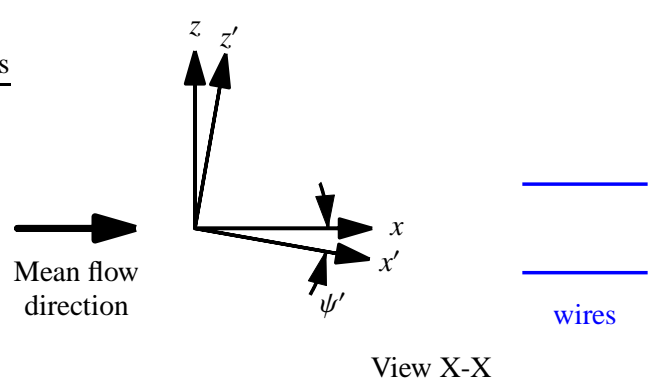

Fig. 10: Relation between the wind-tunnel frame of reference $x-y-z$, and the adopted frame of reference for the twodimensional calibration, $x^{\prime}-y^{\prime}-z^{\prime}$; shown for an $\times$-probe configured to measure the $u$ and $v$ velocities. The angles in (a) $\phi^{\prime}$ and (b) $\psi^{\prime}$ correspond to in-plane and out-of-plane misalignment respectively, between the two frames of reference with respect to the sensor plane.

rection in the wind-tunnel (see figure 10), leading to inaccuracy in the velocity-voltage mapping function constructed. To mitigate this error, a second calibration is performed in the wind tunnel where a zero-angle calibration is repeated. Thus, angular misalignment present during the two-dimensiona calibration could be corrected for by rotating the jet frame of reference until the two zero-angle calibration match. It should be noted that, this procedure requires the velocityvoltage relationship of the $x$-probe to not change between the two calibration, and any change in the calibration functional form due to drift in ambient or hot-wire properties will lead to erroneous corrections to be applied.

The misalignment between the two frames of reference can be described in terms of two orthogonal rotations about the $y$ and $z$ axes. The same misalignment can also be described in terms of angles with respect to the sensor plane, $\phi^{\prime}$ and $\psi^{\prime}$, as shown in figures $10(a)$ and $(b)$. The former corresponds to misalignment in the sensor plane formed by the two-wires, while the latter corresponds to misalignment in the normal direction to the sensor plane. Therefore, the velocities in the calibration and wind-tunnel frame of reference can be related as

$u=\cos \psi^{\prime}\left(u^{\prime} \cos \phi^{\prime}-v^{\prime} \sin \phi^{\prime}\right)$,

$v=\cos \psi^{\prime}\left(u^{\prime} \sin \phi^{\prime}+v^{\prime} \cos \phi^{\prime}\right)$

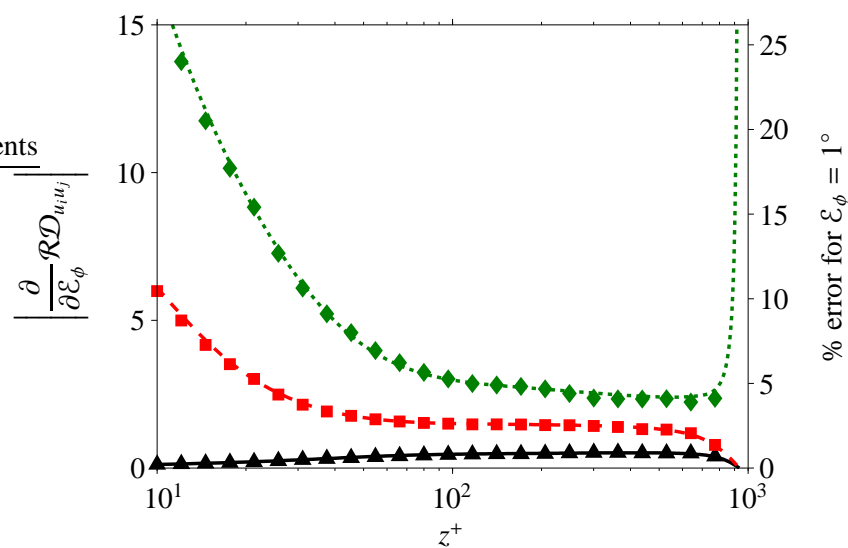

Fig. 11: Sensitivity of the turbulent stresses to the misalignment between the coordinate system employed during the two-dimensional calibration and measurement process. The coefficient of proportionality with respect to the misalignment angle for the relative difference of the $\overline{u^{2}}(\mathbf{\Lambda},-), \overline{w^{2}}$ $(\square,---)$, and $\overline{u w}(\diamond, \cdots)$ statistics from the $u-w \times$-probe. The symbols corresponds to the sensitivity coefficients measured directly using the DNS field, while the lines are evaluated from the turbulent stresses using equation (8).

where $u^{\prime}$ and $v^{\prime}$ indicate velocity components with respect to the frame of reference during calibration, while $u$ and $v$ are velocity components with respect to the wind-tunnel frame of reference. It should be noted that the term $v$ in equation (5) can be replaced with the term $-w$ depending on the configuration of the $\times$-probe used. Therefore, following equation (4), errors in the $u$ and $v$ velocities for small misalignments $\mathcal{E}_{\phi}$ and $\mathcal{E}_{\psi}$, measured in radians, are given by

$\mathcal{E}_{u} \approx-v \mathcal{E}_{\phi}, \quad$ and $\quad \mathcal{E}_{v} \approx u \mathcal{E}_{\phi}$.

Thus, the errors in the turbulence statistics can be expressed for the $u$-v $\times$-probe as

$\mathcal{R D _ { u u }}=-\frac{2 \overline{u v}}{\overline{u^{2}}} \varepsilon_{\phi}+O\left(\varepsilon_{\phi}^{2}\right)+O\left(\varepsilon_{\psi}^{2}\right)$,
$\mathcal{R} \mathcal{D}_{v v}=\frac{2 \frac{u v}{\overline{u v}}}{\overline{v^{2}}} \varepsilon_{\phi}+O\left(\varepsilon_{\phi}^{2}\right)+O\left(\varepsilon_{\psi}^{2}\right)$.

Similarly, for the $u$-w $\times$-probe

$\mathcal{R} \mathcal{D}_{u u}=\frac{2 \overline{u w}}{\overline{u^{2}}} \varepsilon_{\phi}+O\left(\varepsilon_{\phi}^{2}\right)+O\left(\varepsilon_{\psi}^{2}\right)$,

$\mathcal{R} \mathcal{D}_{w w}=-\frac{2 \overline{u w}}{\overline{w^{2}}} \varepsilon_{\phi}+O\left(\varepsilon_{\phi}^{2}\right)+O\left(\varepsilon_{\psi}^{2}\right)$,

$\mathcal{R} \mathcal{D}_{u w}=\frac{-\overline{u^{2}}+\overline{w^{2}}}{\overline{u w}} \varepsilon_{\phi}+O\left(\varepsilon_{\phi}^{2}\right)+O\left(\varepsilon_{\psi}^{2}\right)$

For canonical wall-bounded flows, $\overline{u v}=0$ while $\overline{u w} \neq 0$. Hence, the first order terms disappear from $\mathcal{R D}$ for the $u$ $v \times$-probe, implying they are more robust to misalignment 
during the calibration process than the $u$-w $\times$-probe. Indeed, during the experiments we found the $u-v \times$-probe repeat measurements to be more consistent than the $u$ - $w \times$-probe results. Figure 11 shows the sensitivity of $\mathcal{R} \mathcal{D}_{u u}, \mathcal{R} \mathcal{D}_{w w}$ and $\mathcal{R} \mathcal{D}_{u w}$ to $\mathcal{E}_{\phi}$ at various $z$ locations. To evaluate the symbols in figure 11, the turbulence statistics from the DNS velocity field are re-evaluated for the new coordinate system rotated by $\mathcal{E}_{\phi}$. Excellent agreement is observed between the sensitivity coefficients measured using the DNS velocity field and equation (8) (shown as lines). It should be noted that, Strohl \& Comte-Bellot (1973) have performed an investigation on the sensitivity of Reynolds shear stress to misalignment during the calibration process and obtained a different expression for equation (8c), however this may be due to added simplification possible in the particular flow (a jet flow) they investigated. From figure 11, it is evident that the Reynolds shear stress is the most sensitive to the misalignment errors. Although the error due to misalignment is equal for the $\overline{u^{2}}$ and $\overline{w^{2}}$ statistics from the $u$-w $\times$-probe, since $\overline{u^{2}}>\overline{w^{2}}$ the relative error is significantly higher for the wall-normal turbulence intensity. A similar conclusion is drawn by Zimmerman et al. (2017) for the sensitivity of $u$ - $v$ and $u$ - $w \times$-probes to the yaw and pitch calibration misalignment, respectively.

For a series of $\times$-probe measurements undertaken recently at the University of Melbourne (including the results shown in figure 5 insets) (Baidya, 2016), the procedure of matching the zero-angle calibration allowed the velocity-tovoltage mapping function generated to be corrected within $\pm 0.5^{\circ}$ of the wind-tunnel frame of reference. The $\pm 0.5^{\circ} \mathrm{mi}-$ salignment corresponds to uncertainty in $\overline{u^{2}}, \frac{w^{2}}{}$ and $\overline{u w}$ approximately equalling to $0.5,1$ and $2 \%$ respectively, for regions $z^{+}>100$. This is consistent with our experimental observation where we find the largest variability for $\overline{u w}$ among all turbulent stresses between experiment repeats. It should be noted that below $z^{+}<100$, the uncertainty levels are functions of the $z$ location, and may be significantly higher than the corresponding levels stated for $z^{+}>100$. For example, at $z^{+}=15$ these errors are $0.5,4$ and $10 \%$ for the $\overline{u^{2}}, \overline{w^{2}}$ and $\overline{u w}$ statistics, respectively.

\subsection{The sensitivity to errors in the calibration mapping functions}

In $\S 5.2$ we have assumed that the voltage-to-velocity mapping function, which is expressed by a particular functional form or interpolated from the recorded velocities and voltages, can be perfectly captured utilising the calibration procedure described. However, in practice the recorded velocities and voltages during the calibration procedure have associated uncertainties, thus leading to inferred velocities which are erroneous. To investigate the effect of these uncertainties a simulated $\times$-probe is used, as done in the previous secti- ons. However, here the voltage-to-velocity calibration map is perturbed to mirror the uncertainties present during an actual calibration, and the errors are numerically propagated so that sensitivities of the turbulent stresses to disturbances during calibration procedure can be quantified. Furthermore, an inaccuracy at one of the calibrated points leads not only to an error at a single point in the velocity-voltage mapping function but also the surrounding region, and therefore the error propagated is a function of the step size chosen for the jet velocity and jet angle when performing the calibration. Note, to replicate the experimental conditions, we have chosen a similar step size for the jet velocity and jet angle which correspond to $10 \%$ of the full-scale velocity and $5^{\circ}$, respectively (see figure 2 ).

Another significant source of error that may be introduced for $\times$-probe is when specifying a functional form the calibration data should adhere to. This is because unlike the single normal hot-wires where insignificant differences in the calculated $u$ are observed for the typical functions used to describe the mapping function (Bruun et al., 1988), the results from the $\times$-probe can vary significantly based on the functional form used to describe the mapping function $(\mathrm{Bu}-$ rattini \& Antonia, 2005; Dijk \& Nieuwstadt, 2004). In fact one of the challenges associated with an $\times$-probe calibration procedure is to specify the functional form, as the optimal function form can be probe dependent (Bruun, 1995). Furthermore, many functional forms proposed in the literature are found to be too idealised and not capture the real hotwire responses, leading to substantial errors (up to 50\%) (Dijk \& Nieuwstadt, 2004). Here, we will restrict ourselves to the same calibration and fitting procedures as that utilised for the experiments, and therefore is unable to fully account for bias errors introduced due to selection of an incorrect functional form. Instead, here we aim to quantify the expected spread in the measured statistics, due to uncertainties introduced during the calibration procedure.

Figure 12 shows the error in the turbulent stresses due to a perturbation to the voltages, $\mathcal{E}_{E}$, introduced at a single calibration point located at $U_{\text {jet }}=0.8 U_{\mathrm{CL}}$ and $\phi=5^{\circ}$, where $U_{\text {jet }}$ and $\phi$ corresponds to the jet velocity and jet angle, while $U_{\mathrm{CL}}$ denotes the centreline velocity of the channel DNS. Here, the abscissa and ordinate correspond to the voltage perturbations normalised by the full scale voltage range and the maximum relative difference observed across the entire $z$ location, respectively. Therefore, a $1 \%$ uncertainty in the measured voltage correspond to $1-1.5 \%$ uncertainty in the measured turbulent stresses. Interestingly, while uncertainty in $\overline{u^{2}}, \overline{v^{2}}$ and $\overline{w^{2}}$ show a linear growth with increased uncertainty in the measured voltage, the uncertainty in Reynolds shear stress follows a quadratic behaviour. Furthermore, the sensitivity of each stresses to the perturbation, given by the slope $\partial \mathcal{R} \mathcal{D}_{u_{i} u_{j}} / \partial \mathcal{E}_{E}$, is different and the Reynolds shear stress is observed to grow faster than the other 


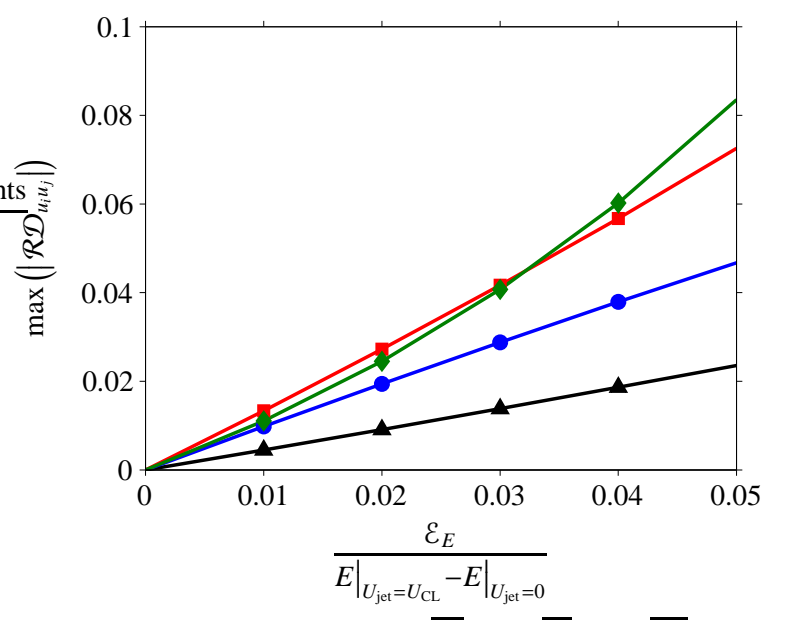

Fig. 12: Relative difference in $\overline{u^{2}}(\mathbf{\Lambda}), \overline{v^{2}}(\bullet), \overline{w^{2}}(\square)$, and $\overline{u w}(\diamond)$ as functions of perturbation to the velocity-voltage mapping function.

turbulent stresses at a high level of $\mathcal{E}_{E}$. These results agree with our experimental observations, where out of all turbulent stresses the Reynolds shear stress is found to exhibit the largest dependency to the choice of functional form used to describe the voltage-to-velocity conversion.

To relate the spread in turbulent stresses to uncertainties in jet velocities, the velocity perturbation are converted to an uncertainty level in voltage following the chain rule. In other words,

$$
\begin{aligned}
& \frac{\overline{u_{i} u_{j}}-\left.\overline{u_{i} u_{j}}\right|_{\text {ideal }}}{\left.\overline{u_{i} u_{j}}\right|_{\text {ideal }}} \approx \frac{\partial \mathcal{R} \mathcal{D}_{u_{i} u_{j}}}{\partial U_{\text {jet }}} \mathcal{E}_{U_{\text {jet }}}+\frac{\partial \mathcal{R} \mathcal{D}_{u_{i} u_{j}}}{\partial E} \mathcal{E}_{E} \\
& \approx \frac{\partial \mathcal{R} \mathcal{D}_{u_{i} u_{j}}}{\partial E_{1}} \frac{\partial E_{1}}{\partial U_{\text {jet }}} \mathcal{E}_{U_{\text {jet }}}+\frac{\partial \mathcal{R} \mathcal{D}_{u_{i} u_{j}}}{\partial E_{2}} \frac{\partial E_{2}}{\partial U_{\text {jet }}} \varepsilon_{U_{\text {jet }}}+\frac{\partial \mathcal{R} \mathcal{D}_{u_{i} u_{j}}}{\partial E} \mathcal{E}_{E} \\
& \approx \frac{\partial \mathcal{R} \mathcal{D}_{u_{i} u_{j}}}{\partial E}\left(2 \frac{\partial E}{\partial U_{\text {jet }}} \varepsilon_{U_{\text {jet }}}+\mathcal{E}_{E}\right)
\end{aligned}
$$

in the vicinity of $\phi=0$, as $\partial \mathcal{R} \mathcal{D}_{u_{i} u_{j}} / \partial E_{1} \approx \partial \mathcal{R} \mathcal{D}_{u_{i} u_{j}} / \partial E_{2} \approx$ $\partial \mathcal{R} \mathcal{D}_{u_{i} u_{j}} / \partial E$. For a typical calibration, $\mathcal{E}_{E} \sim 0.2 \%$ of the full-scale, while $\mathcal{E}_{U_{\text {jet }}} \sim 1 \%$ and $\partial E / \partial U_{\text {jet }} \sim O(1)$. Thus, the lumped uncertainty corresponding to the terms inside the bracket in equation (9) is equivalent to $\pm 2.5 \%$ uncertainty in the hot-wire voltages. Reading off the $\max \left(\left|\mathcal{R} \mathcal{D}_{u_{i} u_{j}}\right|\right)$ from figure 12 at $\mathcal{E}_{E} /\left(\left.E\right|_{U_{\mathrm{jet}}=U_{\mathrm{CL}}}-\left.E\right|_{U_{\mathrm{jet}}=0}\right)=0.025$, uncertainty of $1,2,3$ and $3 \%$ in the $\overline{u^{2}}, \overline{v^{2}}, \overline{w^{2}}$ and $\overline{u w}$ statistics is expected due to uncertainties associated with measuring the flow velocities and hot-wire voltages during the calibration procedure.

\section{Summary and conclusions}

The effects of measured turbulent stress to uncertainties in the probe geometry and calibration procedure is investigated for a cross-wire probe in a wall-bounded flow. In an experimental study, the uncertainties in probe geometry and calibration procedure cannot be eliminated since intrinsic limitations exist in the manufacturing and calibration processes.

We showed that a misalignment between the wires in the wall-normal direction for a cross-wire probe configured to measure $u$ and $w$ velocities can lead to incorrect $W$ and $\overline{u w}$ statistics, particularly in the near-wall region while the $U, \overline{u^{2}}$ and $\overline{w^{2}}$ statistics remained relatively robust. For a cross-wire probe configured to measure $u$ and $v$ velocities, by design a wall-normal separation much larger than the typical misalignment observed exists (effects of which are discussed in Philip et al. (2013b)), hence the additional error from the misalignment remains negligible.

In addition to uncertainties in wire position, we also consider a misalignment of the wire angular orientation for the cross-wire probe. We find that the misalignment in orientation becomes an important issue when a cross-wire probe is inclined to allow near-wall access. Without compensating for the inclination angle when the cross-wire probe is constructed (this flexibility is not present if using a generic commercial probe), a substantial error, up to $10 \%$, can be introduced for the Reynolds shear stress. Comparison of simulation results against experiments suggests that effects from finite sensor dimensions dominate the attenuation/amplification of $u, v$ and $w$ variances recorded by $\times$ probe, while the Reynolds shear stress is more sensitive to $\times$-probe misalignment.

For uncertainties during the calibration procedure, errors due to misalignment and perturbation to the voltage-tovelocity calibration map is considered. The first scenario can occur when the probes need to be unmounted and remounted between calibration and measurement, which is typically necessary if using a commercially available calibration device. To alleviate the misalignment errors, a calibration jet facility with a pitch and yaw control was used instead to calibrate the cross-wire probe in situ, without unmounting the probe from the sting fixture attached to the wind-tunnel. Our analysis shows that Reynolds shear stress is particularly sensitive (twice or more than the other stresses) to the calibration misalignment errors. This may explain the largest spread occurring for the Reynolds shear stress among the turbulent stresses in our experiment and substantial scatter of Reynolds shear stress evident in the literature, notably in recent high Reynolds number pipe flow measurements (Örlü et al., 2017), and in a compilation of turbulent statistics in boundary layer flows from Fernholz \& Finley (1996). Finally, uncertainties in the measured velocity and voltages during the calibration procedure is numerically propagated through perturbation to the voltage-to-velocity calibration map. The calibration uncertainties lead to a quadratic growth in Reynolds shear stress error, compared to a linear behaviour for $\overline{u^{2}}, \overline{v^{2}}$ and $\overline{w^{2}}$ errors. Hence, Reynolds shear stress error has 
a tendency to get severely amplified when a substantial departure from the correct voltage-to-velocity map occurs due to uncertainties present during the calibration procedure. As such, it is not surprising to see the Reynolds shear stress in wall turbulence exceed the theoretically highest value of unity, when scaled by viscous units (eg. Fernholz \& Finley (1996)). This is consistent with our empirical observations where the Reynolds shear stress is found to be more sensitive than the $\overline{u^{2}}, \overline{v^{2}}$ and $\overline{w^{2}}$ statistics to the functional form employed for the voltage-to-velocity calibration map.

\section{Acknowledgement}

The authors gratefully acknowledge the financial support from the Australian Research Council. The authors would also like to thank Prof. R. D. Moser for making the channel DNS database available.

\section{References}

Baidya, R. 2016 Multi-component velocity measurements in turbulent boundary layers. PhD thesis, University of Melbourne.

Baidya, R., Philip, J., Hutchins, N., Monty, J. P. \& Marusic, I. 2019 Spatial averaging effects on the streamwise and wall-normal velocity measurments in a wall-bounded turbulence using a cross-wire probe. Meas. Sci. Technol. 30, 085303.

Baidya, R., Philip, J., Hutchins, N., Monty, J. P. \& Marusic, I. 2017 Distance-from-the-wall scaling of turbulent motions in wall-bounded flows. Phys. Fluids 29 (2), 020712.

Browne, L. W. B., Antonia, R. A. \& Chua, L. P. 1988 Calibration of $\times$-probes for turbulent flow measurements. Exp. Fluids 7 (3), 201-208.

Bruun, H. H. 1995 Hot-wire anemometry: principles and signal analysis. Oxford Univ Press.

Bruun, H. H., Khan, M. A., Al-Kayiem, H. H. \& Fardad, A. A. 1988 Velocity calibration relationships for hot-wire anemometry. J. Phys. E: Sci. Instrum. 21 (2), 225.

Burattini, P. \& Antonia, R. A. 2005 The effect of different $X$-wire calibration schemes on some turbulence statistics. Exp. Fluids 38 (1), 80-89.

Champagne, F. H., Sleicher, C. A. \& Wehrmann, O. H. 1967 Turbulence measurements with inclined hot-wires Part 1. Heat transfer experiments with inclined hot-wire. J. Fluid Mech. 28 (01), 153-175.

Chin, C., Hutchins, N., Ooi, A. \& Marusic, I. 2011 Spatial resolution correction for hot-wire anemometry in wall turbulence. Exp. Fluids 50 (5), 1443-1453.

del Álamo, J. C., Jiménez, J., Zandonade, P. \& Moser, R. D. 2004 Scaling of the energy spectra of turbulent channels. J. Fluid Mech. 500, 135-144.
Dijk, A. Van \& Nieuwstadt, F. T. M. 2004 The calibration of (multi-) hot-wire probes. 2. Velocity-calibration. Exp. Fluids 36 (4), 550-564.

Fernholz, H. H. \& Finley, P. J. 1996 The incompressible zero-pressure-gradient turbulent boundary layer: an assessment of the data. Prog. Aerosp. Sci. 32 (4), 245-311.

Hutchins, N. \& Choi, K. S. 2002 Accurate measurements of local skin friction coefficient using hot-wire anemometry. Prog. Aerosp. Sci. 38 (4-5), 421-446.

Janke, G. 1987 Hot wire in wall proximity. In Advances in Turbulence, pp. 488-498. Springer.

Jørgensen, F. E. 1996 The computer-controlled constanttemperature anemometer. Aspects of set-up, probe calibration, data acquisition and data conversion. Meas. Sci. Technol. 7 (10), 1378.

King, L. V. 1914 On the convection of heat from small cylinders in a stream of fluid: determination of the convection constants of small platinum wires, with applications to hot-wire anemometry. Philos. Trans. R. Soc. London, Ser. A 90 (622), 563-570.

Ligrani, P. M. \& Bradshaw, P. 1987 Subminiature hot-wire sensors: development and use. J. Phys. E: Sci. Instrum. 20 (3), 323.

Moin, P. \& Spalart, P. R. 1989 Contributions of numerical simulation data bases to the physics, modeling, and measurement of turbulence. Tech. Rep. NASA-TM-100022. NASA.

Örlü, R., Fiorini, T., Segalini, A., Bellani, G., Talamelli, A. \& Alfredsson, P. H. 2017 Reynolds stress scaling in pipe flow turbulence - first results from CICLoPE. Philos. Trans. R. Soc. London, Ser. A 375 (2089), 20160187.

Örlü, R. \& Schlatter, P. 2013 Comparison of experiments and simulations for zero pressure gradient turbulent boundary layers at moderate Reynolds numbers. Exp. Fluids 54 (6), 1547.

Perry, A. E. 1982 Hot-wire anemometry. Oxford Univ Press.

Philip, J., Hutchins, N., Monty, J. P. \& Marusic, I. $2013 a$ Spatial averaging of velocity measurements in wallbounded turbulence: single hot-wires. Meas. Sci. Technol. 24 (11), 115301.

Philip, J., Baidya, R., Hutchins, N., Monty, J. P. \& Marusic, I. $2013 b$ Spatial averaging of streamwise and spanwise velocity measurements in wall-bounded turbulence using $\vee$ - and ×-probes. Meas. Sci. Technol. 24 (11), 115302.

Segalini, A., Cimarelli, A., Rüedi, J-D., De Angelis, E. \& Talamelli, A. 2011 Effect of the spatial filtering and alignment error of hot-wire probes in a wall-bounded turbulent flow. Meas. Sci. Technol. 22 (10), 105408.

Smits, A. J., McKeon, B. J. \& Marusic, I. 2011 HighReynolds number wall turbulence. Annu. Rev. Fluid Mech. 43, 353-375.

Strohl, A. \& Comte-Bellot, G. 1973 Aerodynamic effects due to configuration of $\times$-wire anemometers. J. Appl. 
Mech. 40 (3), 661-667.

Suzuki, Y. \& Kasagi, N. 1992 Evaluation of hot-wire measurements in wall shear turbulence using a direct numerical simulation database. Exp. Therm Fluid Sci. 5 (1), 69-77.

Talamelli, A., Westin, K. J. A. \& Alfredsson, P. H. 2000 An experimental investigation of the response of hot-wire $\times$ probes in shear flows. Exp. Fluids 28 (5), 425-435.

Talluru, K. M., Kulandaivelu, V., Hutchins, N. \& Marusic, I. 2014 A calibration technique to correct sensor drift issues in hot-wire anemometry. Meas. Sci. Technol. 25 (10), 105304.

Yavuzkurt, S. 1984 A guide to uncertainty analysis of hotwire data. J. Fluid Eng. 106 (2), 181-186.

Zhao, R., Li, J. \& Smits, A. 2004 A new calibration method for crossed hot wires. Meas. Sci. Technol. 15 (9), 1926.

Zimmerman, S., Morrill-Winter, C. \& Klewicki, J. 2017 Design and implementation of a hot-wire probe for simultaneous velocity and vorticity vector measurements in boundary layers. Exp. Fluids 58 (10), 148. 